nephron

Experimental

Nephrology

and Genetics
Nephron 2015;129:293-299

DOI: $10.1159 / 000377633$
Received: October 27, 2014

Accepted after revision: January 31, 2015

Published online: March 10, 2015

\title{
Serum Klotho: Relation to Fibroblast Growth Factor-23 and Other Regulators of Phosphate Metabolism in Children with Chronic Kidney Disease
}

\author{
Happy K. Sawires ${ }^{a}$ Rasha M. Essam ${ }^{a}$ Marian F. Morgan ${ }^{b}$ Rasha A. Mahmoud ${ }^{c}$ \\ Departments of a Pediatrics and ${ }^{b}$ Chemical Pathology, Cairo University, and ${ }^{c}$ Ministry of Health, Cairo, Egypt
}

\begin{abstract}
Key Words
FGF-23 · Klotho · Phosphorus - Chronic kidney disease . Kidney transplantation · Dialysis
\end{abstract}

\begin{abstract}
FGF23 and Klotho synergize to regulate phosphate homeostasis by promoting renal phosphate excretion. Chronic kidney disease (CKD) may be viewed as a state of FGF23 resistance caused by Klotho deficiency. This viewpoint explains several observations on phosphate metabolism in CKD that lack mechanistic insights. Our objectives were to correlate serum klotho and FGF-23 with other variables that regulate phosphate metabolism. We studied 40 patients with CKD on conservative treatment (group A), 44 patients with endstage renal disease (ESRD) on regular hemodialysis (group B), 40 kidney transplant recipients (KTR) (group C) and 40 healthy controls for measuring serum klotho and FGF-23. Blood samples were withdrawn for measuring the levels of serum Calcium (Ca), Phosphorus ( $\mathrm{P})$, alkaline phosphatase (ALP), 1,25(OH) ${ }_{2} D_{3}$, intact parathyroid hormone (PTH), FGF23 and a klotho. The mean levels of FGF-23 and a klotho in control group were $225.78 \pm 111.05 \mathrm{pg} / \mathrm{ml}$ (range: 102.4, 418.5 ) and $6.78 \pm 1.90 \mathrm{ng} / \mathrm{ml}$ (range: 4,11 ), respectively. The mean levels of FGF-23 in the 3 studied groups were 1,034.2 \pm $84.6,1,288.7 \pm 131.4$ and $1,008.7 \pm 117.6 \mathrm{pg} / \mathrm{ml}$, respectively. The median levels of s-klotho in the 3 studied groups were $3.15,2.3$ and 2.95, respectively. It was found that FGF-23 was significantly increased and a klotho was significantly de-
\end{abstract}

creased in all patients when compared with those in the control group ( $p<0.001,<0.001$, respectively). We found that there was a significant inverse correlation between serum Ca and a klotho in the studied groups. There was no significant correlation between FGF-23 and a klotho in the studied groups ( $p>0.05$ ). We have shown that circulating s-klotho was not related to FGF-23 in CKD, dialysis and KTR patients. In addition, we demonstrated a novel association between serum Ca and s-klotho that needs to be further studied.

(c) 2015 S. Karger AG, Basel

\section{Introduction}

The klotho gene, name refers to a Greek goddess who spins the thread of life, was originally identified as a mutated gene in a mouse strain that leads to the prematureaging syndrome in an autosomal recessive inheritance [1].

Klotho results either from direct secretion by the cell or from cleavage of the intracellular domain of the full length protein by secretases. Both processes lead to 'soluble Klotho,' which is found in blood, urine, and cerebrospinal fluid [26].

The kidney and the parathyroid are the main organs that express klotho. In humans, klotho polymorphisms have been associated with bone mineral density, cardiovascular events (e.g., carotid atherosclerosis, ischemic stroke), life expectancy, biomarkers of metabolic syn-

\section{KARGER 125}

(c) 2015 S. Karger AG, Base

$1660-8151 / 15 / 1294-0293 \$ 39.50 / 0$

E-Mail karger@karger.com

www.karger.com/nef
Prof. Dr. Happy K. Sawires

Pediatric Department, Cairo University

5 El-Lithy Street El-Maadi

El-Gedida, Cairo (Egypt)

E-Mail Happy7_kd@yahoo.com 
drome (e.g., uric acid levels, lipid and glucose metabolisms), and even with cognitive ability [2].

Klotho expression in kidney and parathyroid gland identifies these organs as high-affinity fibroblast growth factor 23 (FGF-23) endocrine targets. Thus, the Klotho/ FGFR complex mediates FGF-23 participation in the bone - kidney - parathyroid endocrine axis. In the kidneys, FGF-23, acting on Klotho/FGFR, suppresses phosphate reabsorption and $1,25(\mathrm{OH})_{2} \mathrm{D}_{3}$ synthesis; in the parathyroid, FGF-23 suppresses parathyroid hormone secretion [3].

Thus, FGF23 and Klotho synergize to regulate phosphate homeostasis by enhancing renal phosphate excretion: they do so through the reduction of the number of $\mathrm{NaPiIIa}$ and NaPiIIc phosphate co-transporters in the proximal renal tubule and reduction of absorption of intestinal phosphate, the latter following a decreased renal synthesis of $1,25(\mathrm{OH})_{2} \mathrm{D}_{3}$ [4].

It is likely that FGF23-Klotho endocrine axis dysregulation may be involved in the mechanism by which chronic kidney disease (CKD) patients fail to maintain phosphate homeostasis. Thus, CKD may be viewed as a state of FGF23 resistance caused by relative Klotho deficiency. This viewpoint explains numerous observations on phosphate metabolism in CKD that lack mechanistic insights [5].

We determined the serum level of klotho (which reflects klotho gene expression) and FGF-23 in a crosssectional study in CKD, dialysis and renal transplant patients. Our objectives were to correlate serum klotho and FGF-23 with other variables that regulate phosphate metabolism and to find the best indicator of serum klotho.

\section{Methodology}

We studied 40 patients with CKD stage $2-5$ on conservative treatment, 44 patients with ESRD who were stable on regular hemodialysis for at least 3 months prior to the study, 40 kidney transplant recipients (KTR) and 40 healthy controls. This study included patients of both sexes and different ages. The patients were recruited from the Outpatient Nephrology Clinic and Centre of Pediatric Nephrology and Transplantation (CPNT), Cairo University. Age- and sex-matched control subjects (23 males and 17 females with mean age $8.3 \pm 3.74$ years) who came for check-up were recruited from the outpatient general pediatric clinics for measuring the serum levels of klotho and FGF-23.

The following patients were excluded from the study: those with marked hypocalcemia (total serum calcium $<7 \mathrm{mg} / \mathrm{dl}$ or ionized calcium $<1 \mathrm{mmol} / \mathrm{l}$ ), with unsatisfactory vascular access affecting dialysis adequacy, with combined organ transplantation, those who had total or subtotal surgical parathyroidectomy and those patients in whom the original disease was associated with an overproduction of vitamin D (1 patient was excluded because of sarcoidosis).

Vitamin D and calcium (Ca) supplementations, phosphate binders or calcimimetics were stopped 30 days prior to enrollment in the study. An informed written consent was taken from the patients' care providers before the study.

Patients were classified into 3 groups: group A: CKD patients, group B: ESRD patients on regular dialysis and group C: KTR.

The patients were enrolled in a cross-sectional study. The following data were collected from all patients: age, gender, original disease, medications that may affect $\mathrm{Ca}$ and phosphorus (P) levels, duration of dialysis for dialysis patients and duration of transplantation for KTR.

The Schwartz formula was used to calculate eGFR, while Kt/V was used to assess dialysis adequacy.

For all patients, blood samples were withdrawn (for hemodialysis (HD) patients; they were collected immediately after placing the dialysis needle and thus before start of dialysis in the middle of the week) for measuring the levels of serum $\mathrm{Ca}, \mathrm{P}$, alkaline phosphatase (ALP), 1,25 (OH $)_{2} \mathrm{D}_{3}$, intact parathyroid hormone (PTH), FGF-23 and $\alpha$ klotho.

PTH was measured by immunoradiometric assay using Elecsys 2010 autoanalyzer system, Roche Diagnostics, Germany. Serum $\mathrm{Ca}, \mathrm{P}$ and ALP were measured using the Synchron cx5 autoanalyzer, Beckman, USA.

Serum FGF-23 concentrations were measured using a secondgeneration, two-site, monoclonal antibody ELISA kit according to the manufacturer's procedure (Kainos Laboratories, Tokyo, Japan).

Serum klotho was measured by human Klotho ELISA kit, Catalogue number E2253h supplied from EIAab.www.eiab.com. The microtitre plate provided by the kit had been coated with an antibody specific to Klotho. Standards or samples were then added to the appropriate microtitre plate with a biotin-conjugated polyclonal antibody preparation specific for Klotho, and Avidin conjugated to the Horseraddish peroxidase was added to each microplate well and incubated. Then a TMB substrate solution was added to each well. Only those wells that contain Klotho, biotinconjugated antibody and enzyme-conjugated Avidin exhibited a change in color. The enzyme - reaction was terminated by the addition of a sulfuric acid solution and the color change was measured spectrophotometrically at W.L. of $450 \mathrm{~nm}$. The concentration of Klotho in the sample was then determined by comparing the O.D. of the samples to the standard curve.

\section{Statistical Methods}

Quantitative parametric data were presented as mean and standard deviation, while quantitative nonparametric data were presented as median and inter-quartile range (IQR). Qualitative (Categorical) data were presented as frequencies and percentages. For multiple comparisons of several groups, ANOVA or the KruskalWallis test were performed. The Chi-square test was used to compare qualitative variables. The Spearman correlation coefficient was used to determine significant correlations between nonlinear quantitative data. Multivariate Linear regression analysis models were used to test for the preferential effect of the independent variable(s) on klotho level. The significance level was set at $\mathrm{p}<0.05$. Statistical analysis was performed with SPSS 16.0 (statistical package for scientific studies) for windows. 
Table 1. Characteristics of the studied patients

\begin{tabular}{|c|c|c|c|c|}
\hline Age* & $3.5(1.5,12.25)$ & $7.5(5,10)$ & $10(8.25,13)$ & $<0.001$ \\
\hline \multicolumn{5}{|l|}{ Gender, $\mathrm{n}(\%)^{\ddagger}$} \\
\hline Male & $28(70)$ & $20(45.5)$ & $24(60)$ & \\
\hline Female & $12(30)$ & $24(54.5)$ & $16(40)$ & 0.072 \\
\hline \multicolumn{5}{|l|}{ Original disease, $\mathrm{n}(\%)^{\ddagger}$} \\
\hline TIN & $4(10)$ & $10(22.7)$ & $8(20)$ & \\
\hline $\mathrm{NPH}$ & $4(10)$ & $4(9.1)$ & $8(20)$ & \\
\hline Cystinosis & $8(20)$ & $2(4.6)$ & $2(5)$ & \\
\hline FSGS & $6(15)$ & $0(0)$ & $2(5)$ & \\
\hline Others & $4(10)$ & $6(13.6)$ & $6(15)$ & \\
\hline Creatinine, $\mathrm{mg} / \mathrm{dl}^{\dagger}$ & $2.61 \pm 1.47$ & $4.89 \pm 0.79$ & $0.85 \pm 0.14$ & $<0.001$ \\
\hline PTH, pg/ml* & $876.2(556.3,1,338.9)$ & $1,873.7(1,177.6,2,114)$ & $855.5(697,1,014.01)$ & $<0.001$ \\
\hline $1,25(\mathrm{OH})_{2} \mathrm{D}_{3}, \mathrm{pmol} / \mathrm{l}^{\dagger}$ & $29.63 \pm 3.71$ & $25.59 \pm 3.28$ & $33.13 \pm 3.52$ & $<0.001$ \\
\hline FGF-23, pg/ml ${ }^{\dagger}$ & $1,034.2 \pm 84.6$ & $1,288.7 \pm 131.4$ & $1,008.7 \pm 117.6$ & $<0.001$ \\
\hline a klotho, ng/ml* & $3.15(2.15,4.27)$ & $2.3(1.9,3.1)$ & $2.95(0.98,4.15)$ & 0.086 \\
\hline
\end{tabular}

ALP = Alkaline phosphatase; $\mathrm{eGFR}=$ estimated glomerular filtration rate; FSGS $=$ focal segmental glomerulosclerosis; $\mathrm{NPH}=$ nephronophthisis; PTH = parathyroid hormone; TIN = tubule-interstitial nephritis. ${ }^{*}$ Kruskal-Wallis test was used, ${ }^{\dagger}$ Anova test was used, ${ }^{\ddagger}$ Chisquare test was used.

\section{Results}

Out of the 40 studied CKD patients, there were 4 patients (10\%) CKD stage 2, 8 patients (20\%) stage 3, 20 patients (50\%) stage 4 and 8 patients (20\%) stage 5 still under conservative treatment awaiting either dialysis or transplantation. The mean duration of dialysis was $2.28 \pm 0.37$ years $(\min .=1$ and $\max .=5)$, while the mean duration after transplantation was $2.22 \pm 0.95$ years ( $\mathrm{min} .=1$ and max. $=4$ ). The mean Kt/V was $1.78 \pm 0.62$. Other characteristics of the studied patients are shown in table 1.

The mean levels of FGF-23 and a klotho in the control group were $225.78 \pm 111.05 \mathrm{pg} / \mathrm{ml}$ (range: $102.4,418.5$ ) and $6.78 \pm 1.90 \mathrm{ng} / \mathrm{ml}$ (range: 4,11 ), respectively.

It was found that FGF-23 was significantly increased and a klotho was significantly decreased in all patients when compared with those in the control group ( $\mathrm{p}<$ $0.001,<0.001$, respectively). Correlations between FGF23 and a klotho with different parameters are shown in table 2 and figure 1.

We found that there was an inverse significant correlation between serum $\mathrm{Ca}$ and $\alpha$ klotho in the studied groups
A, B and C (fig. 2) ( $\mathrm{p}=0.016,0.019$ and 0.001 , respectively).

By using multivariate regression analysis to detect the independent predictors of klotho and FGF-23, we found that only $\mathrm{Ca}$ was an independent predictor for klotho in group $\mathrm{A}$, while both eGFR and PTH were predictors of FGF-23 in the same group. In group B, age and Ca were predictors of klotho, while the duration of dialysis, $\mathrm{Ca}, \mathrm{P}$ and $\mathrm{PTH}$ were independent predictors of FGF-23. In group $\mathrm{C}$, age, $\mathrm{Ca}, \mathrm{P}, \mathrm{FGF}-23$ and $1,25(\mathrm{OH})_{2} \mathrm{D}_{3}$ were the independent predictors of klotho, while $\mathrm{Ca}, \mathrm{PTH}$ and klotho were predictors of FGF-23 (table 3).

\section{Discussion}

Recent identification of FGF-23 and its co-receptor klotho has modified our understanding of derangements of calcium-phosphate metabolism in CKD patients [6]. In this study, in agreement with many studies [7-10], we found that FGF-23 was significantly increased and a klotho was significantly decreased in CKD, dialysis pa- 
Table 2. Correlations between FGF-23 and klotho with different parameters

\begin{tabular}{|c|c|c|c|c|c|c|}
\hline & \multicolumn{2}{|c|}{ Group A $(\mathrm{n}=40)$} & \multicolumn{2}{|c|}{ Group B $(\mathrm{n}=44)$} & \multicolumn{2}{|c|}{ Group C $(\mathrm{n}=40)$} \\
\hline & FGF-23 & a klotho & FGF-23 & a klotho & FGF-23 & a klotho \\
\hline \multicolumn{7}{|l|}{ Age } \\
\hline $\mathrm{r}$ & -0.061 & -0.17 & -0.14 & $-0.30^{*}$ & -0.251 & $-0.495^{*}$ \\
\hline $\mathrm{p}$ & 0.71 & 0.915 & 0.366 & 0.048 & 0.118 & 0.001 \\
\hline \multicolumn{7}{|c|}{ Creatinine } \\
\hline $\mathrm{r}$ & $-0.7^{*}$ & -0.75 & $-0.34^{*}$ & -0.006 & $-0.313^{*}$ & $-0.449^{*}$ \\
\hline $\mathrm{p}$ & $<0.001$ & 0.645 & 0.022 & 0.971 & 0.050 & 0.004 \\
\hline \multicolumn{7}{|l|}{ eGFR } \\
\hline $\mathrm{r}$ & $-0.89^{*}$ & -0.11 & -0.1 & -0.29 & $-0.38^{*}$ & -0.11 \\
\hline $\mathrm{p}$ & $<0.001$ & 0.518 & 0.509 & 0.056 & 0.016 & 0.52 \\
\hline \multicolumn{7}{|c|}{ Duration of dialysis } \\
\hline $\mathrm{r}$ & - & - & $-0.54^{*}$ & -0.65 & - & - \\
\hline $\mathrm{p}$ & & & $<0.001$ & 0.673 & & \\
\hline \multicolumn{7}{|l|}{$\mathrm{Kt} / \mathrm{V}$} \\
\hline $\mathrm{r}$ & - & - & -0.241 & -0.13 & - & - \\
\hline $\mathrm{p}$ & & & 0.115 & 0.399 & & \\
\hline \multicolumn{7}{|c|}{ Duration of transplantation } \\
\hline $\mathrm{r}$ & - & - & - & - & $-0.78^{*}$ & -0.07 \\
\hline $\mathrm{p}$ & & & & & $<0.001$ & 0.666 \\
\hline \multicolumn{7}{|l|}{$\mathrm{Ca}$} \\
\hline $\mathrm{r}$ & $-0.32 *$ & $-0.082^{*}$ & $-0.47^{*}$ & $-0.35^{*}$ & $-0.68^{*}$ & $-0.51^{*}$ \\
\hline $\mathrm{p}$ & 0.044 & 0.016 & 0.001 & 0.019 & $<0.001$ & 0.001 \\
\hline \multicolumn{7}{|l|}{$\mathrm{P}$} \\
\hline $\mathrm{r}$ & $-0.77^{*}$ & -0.245 & $-0.70^{*}$ & -0.83 & $-0.99^{*}$ & -0.012 \\
\hline $\mathrm{p}$ & $<0.001$ & 0.128 & $<0.001$ & 0.593 & $<0.001$ & 0.941 \\
\hline \multicolumn{7}{|l|}{ ALP } \\
\hline $\mathrm{r}$ & -0.25 & $-0.33^{*}$ & -0.128 & -0.152 & -0.004 & -0.146 \\
\hline $\mathrm{p}$ & 0.879 & 0.038 & 0.409 & 0.323 & 0.982 & 0.368 \\
\hline \multicolumn{7}{|l|}{ PTH } \\
\hline$r$ & $-0.89^{*}$ & -0.227 & $-0.91^{*}$ & -0.149 & $-0.36^{*}$ & $-0.36^{*}$ \\
\hline $\mathrm{p}$ & $<0.001$ & 0.158 & $<0.001$ & 0.335 & 0.024 & 0.022 \\
\hline \multicolumn{7}{|c|}{$1,25(\mathrm{OH})_{2} \mathrm{D}_{3}$} \\
\hline $\mathrm{r}$ & $-0.91^{*}$ & -0.161 & $-0.81^{*}$ & -0.054 & $-0.35^{*}$ & $-0.33^{*}$ \\
\hline $\mathrm{p}$ & $<0.001$ & 0.321 & $<0.001$ & 0.726 & 0.025 & 0.034 \\
\hline \multicolumn{7}{|c|}{ FGF-23 } \\
\hline $\mathrm{r}$ & - & -0.286 & - & -0.155 & - & -0.039 \\
\hline $\mathrm{p}$ & & 0.074 & & 0.316 & & 0.810 \\
\hline \multicolumn{7}{|c|}{ a klotho } \\
\hline $\mathrm{r}$ & -0.286 & - & -0.155 & - & -0.039 & - \\
\hline $\mathrm{p}$ & 0.074 & & 0.316 & & 0.810 & \\
\hline
\end{tabular}

tients and even after renal transplantation when compared with those in the control group. CKD may be viewed as a state of FGF-23 resistance caused by the deficiency of klotho. This viewpoint explains why CKD patients fail to maintain phosphate homeostasis [2]. After renal transplantation, high FGF-23 accounts for hypo-

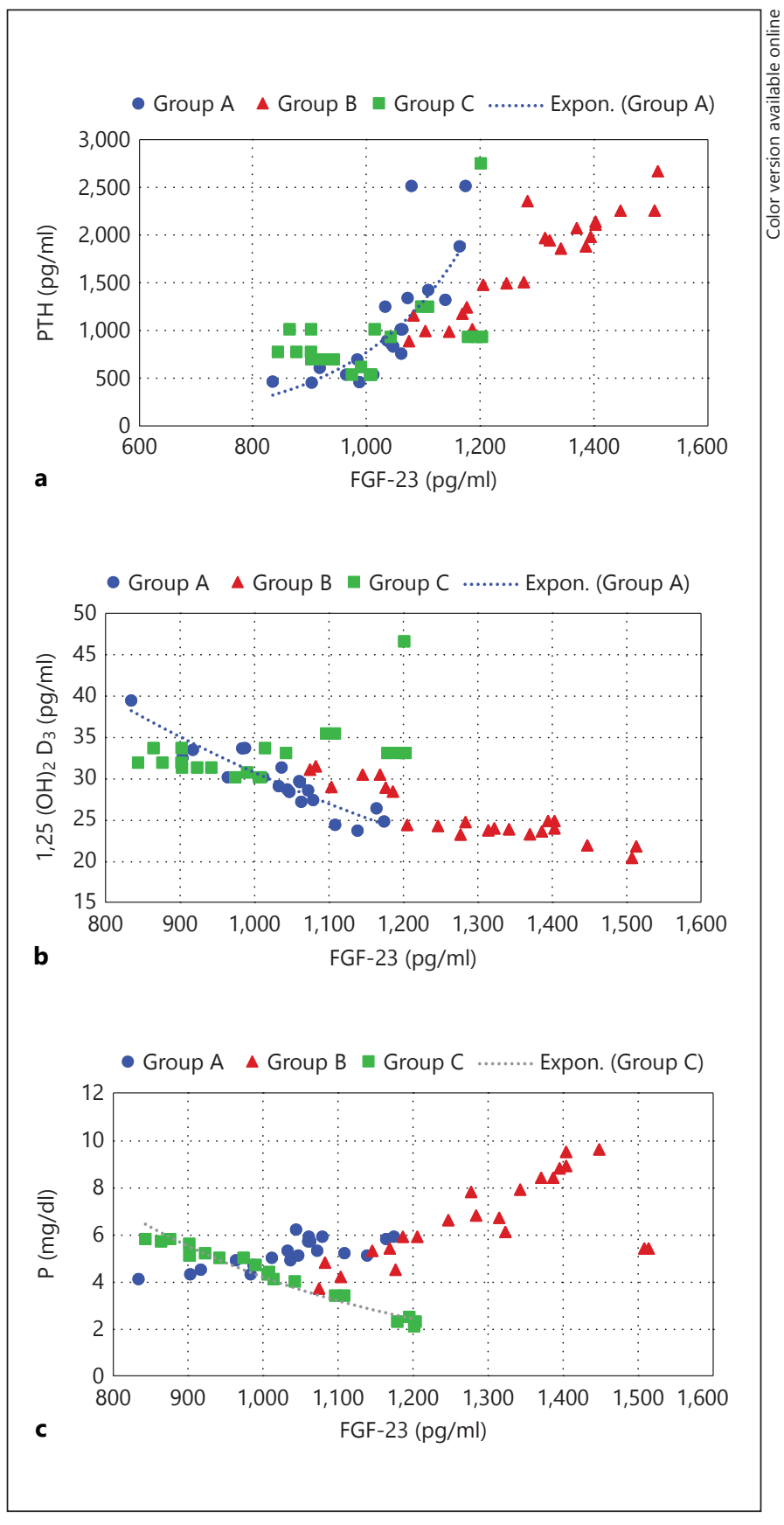

Fig. 1. Correlations of FGF-23 with (a) PTH, (b) $1,25(\mathrm{OH})_{2} \mathrm{D}_{3}$ and (c) $\mathrm{P}$ in different studied groups.

phosphatemia and this negative correlation was observed in our study in agreement with Bhan et al. [11]. Also, this is supported by our observation that the longer the duration after transplantation was, the lower was the level of FGF-23 and hence phosphate level rises towards normal values. 
In CKD patients, it was found that as eGFR decreased, FGF-23 levels increased but this was not in parallel with decline in a klotho. However, according to our results, this correlation was not observed in HD patients. Most of studies show a progressive rise of FGF-23 with a decline of eGFR [12,13], but other studies give discrepant results about serum klotho (s-klotho) and CKD stages.

Sugiura and colleagues reported an elevation of s-klotho in 30 elderly CKD patients when compared to 10 healthy volunteers [14]. On the other hand, Pavik et al. showed a progressive decrease of s-klotho with increasing CKD stage [15]. A significant reduction of s-klotho has been shown in HD patients $[16,17]$. In a recent large cohort study to determine the correlation between s-klotho and different CKD stages, Seiler et al. did not find a significant difference of s-klotho concentrations between CKD stage 2, 3a, 3b and 4 patients [6]. The process of measuring s-Klotho has methodological inadequacies, and quantification of membrane-bound Klotho on FGF23 target cells would entail invasive diagnostic procedures, which is not achievable in large studies.

It is worthy of mention that membrane-anchored proteases releases membrane-bound Klotho into plasma, yielding s-Klotho, which can be measured in large-scale cohort studies [6].

In $\mathrm{CKD}$ and $\mathrm{HD}$ patients, FGF-23 correlated positively to $\mathrm{P}$ and $\mathrm{PTH}$ and correlated negatively with $1,25(\mathrm{OH})_{2}$ $\mathrm{D}_{3}$. Several studies showed that higher FGF23 was consistently associated with lower eGFR, higher serum phosphate, higher fractional excretion of phosphate, and lower levels of 1,25-dihydroxyvitamin D independent of eGFR $[12,13]$. The latter finding suggested that the primary mechanism of reduced 1,25-dihydroxyvitamin D levels in progressive CKD was inhibition by FGF23, rather than insufficient renal mass [5]. In patients with ESRD undergoing dialysis, FGF23 rises over time and often reaches levels that are $>1,000$-fold above normal [18]; this finding, which is in agreement with our results, renders ESRD the clinical setting in which the highest levels of FGF23 have been reported.

We found that a klotho decreased with age in ESRD on regular dialysis and KTR but not in CKD patients. Age-dependent change in s-klotho levels has been previously reported in healthy subjects [19] as well as in pediatric CKD population [20]. Lack of homogenous pattern of age in the different studied groups may explain the absence of this correlation in CKD group.

We observed that in KTR, a klotho levels were higher than their levels in ESRD patients but still significantly lower than their levels in healthy volunteers. Different

FGF23 Is Not Related to Serum Klotho in Children with CKD

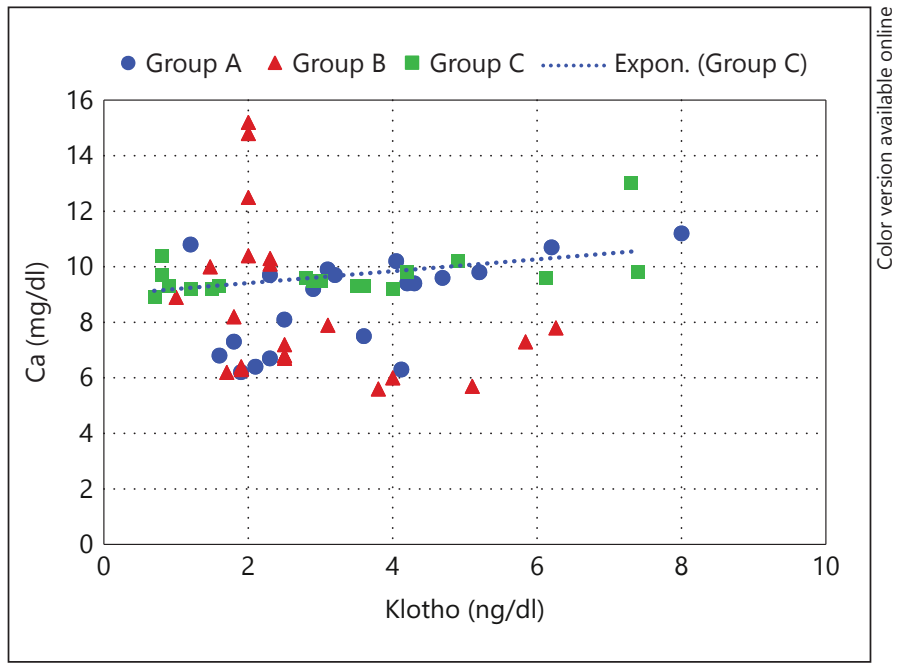

Fig. 2. Correlations of serum klotho and calcium in different studied groups.

Table 3. Multivariate regression analysis to detect predictor of klotho and FGF-23

\begin{tabular}{|c|c|c|c|c|}
\hline & \multicolumn{2}{|c|}{ log klotho } & \multicolumn{2}{|l|}{ FGF-23 } \\
\hline & $\beta$ & $\mathrm{p}$ & $\beta$ & $\mathrm{p}$ \\
\hline \multicolumn{5}{|l|}{ Group A } \\
\hline Age & 0.034 & 0.893 & -0.103 & 0.081 \\
\hline eGFR & 0.044 & 0.951 & -0.709 & $<0.001$ \\
\hline $\mathrm{Ca}$ & -0.109 & 0.034 & -0.024 & 0.690 \\
\hline $\mathrm{P}$ & -0.214 & 0.454 & 0.119 & 0.111 \\
\hline PTH & -0.343 & 0.419 & 5.205 & $<0.001$ \\
\hline $1,25(\mathrm{OH})_{2} \mathrm{D}_{3}$ & 0.053 & 0.928 & 0.769 & 0.447 \\
\hline FGF-23 & 0.218 & 0.771 & - & - \\
\hline Klotho & - & - & 0.010 & 0.853 \\
\hline \multicolumn{5}{|l|}{ Group B } \\
\hline Age & -0.629 & 0.011 & -0.008 & 0.919 \\
\hline Duration of dialysis & 0.170 & 0.479 & -0.260 & 0.001 \\
\hline eGFR & 0.069 & 0.710 & 0.066 & 0.221 \\
\hline $\mathrm{Ca}$ & -0.668 & 0.001 & -0.164 & 0.009 \\
\hline $\mathrm{P}$ & -0.203 & 0.441 & 0.294 & 0.003 \\
\hline PTH & 0.323 & 0.544 & 0.799 & $<0.001$ \\
\hline $1,25(\mathrm{OH})_{2} \mathrm{D}_{3}$ & -0.615 & 0.168 & 0.025 & 0.872 \\
\hline FGF-23 & -0.300 & 0.597 & - & - \\
\hline Klotho & - & - & -0.045 & 0.340 \\
\hline \multicolumn{5}{|l|}{ Group C } \\
\hline Age & -0.416 & 0.019 & -0.024 & 0.431 \\
\hline Duration of & & & & \\
\hline transplantation & 0.148 & 0.448 & 0.021 & 0.528 \\
\hline eGFR & -0.130 & 0.388 & -0.014 & 0.586 \\
\hline $\mathrm{Ca}$ & -0.392 & 0.045 & 0.070 & 0.073 \\
\hline $\mathrm{P}$ & -3.064 & 0.003 & -1.045 & $<0.001$ \\
\hline PTH & -0.292 & 0.237 & -0.086 & 0.036 \\
\hline FGF-23 & -2.882 & 0.003 & - & - \\
\hline Klotho & - & - & -0.089 & 0.003 \\
\hline
\end{tabular}

$1,25(\mathrm{OH})_{2} \mathrm{D}_{3}$ was excluded from analysis in group $\mathrm{C}$. 
studies failed to reach a definitive conclusion about s-klotho levels in KTR. In a recent study, Leone et al. found that KTR had higher s-klotho levels than healthy subjects [21]. On the other hand, Akimoto et al. found low levels of s-klotho after renal transplantation [22]. Experimental studies demonstrated that recombinant human erythropoietin treatment mitigates klotho reduction caused by renal damage [23]. Also, immunosuppressive protocols might modulate the release of s-Klotho from the grafts into the circulation [22]. So, s-klotho should be measured in the context with many other factors that may affect its level.

In univariate analysis, we did not find significant correlation between FGF-23 and a klotho in the studied groups. Only in the KTR group, by multivariate analysis, FGF-23 was an independent predictor of $\alpha$ klotho. The current data about the correlation between FGF-23 and s-klotho are inconclusive. Yamazaki et al. found an inverse correlation between $\alpha$ Klotho and FGF23 in a study performed on 142 healthy volunteers [20]. In another study carried out on $100 \mathrm{HD}$ patients, there was a negative correlation between log FGF23 and log a klotho [24]. On the other hand, Seiler et al. [6] failed to demonstrate any correlation between FGF-23 and a klotho in their univariate analysis. Also, Wan et al. [20] found that log $25(\mathrm{OH}) \mathrm{D}$ was the only significant independent predictor of s-klotho levels in multivariate analysis in a study performed on $154 \mathrm{CKD}$ patients. This contradiction in studies may be attributed to 2 reasons. First, the study performed by Yamazaki et al. was carried out on healthy volunteers and this explains why we found that FGF-23 was an independent predictor of klotho after renal transplantation and return of kidney functions to normal. Second, the currently available s-klotho assays measure soluble s-klotho that may not reflect the molecule in its membrane-bound state. The relationship between circulating and membrane-bound s-klotho, as well as the interaction between circulating s-klotho and FGF23 need to be explored.

We have shown a novel association between serum $\mathrm{Ca}$ levels and increased levels of a klotho in the different studied groups. Huang and Moe demonstrated novel features of klotho include its autocrine-paracrine-endocrine glycan-modifying enzymatic function in the urinary lumen on calcium and phosphate transporters. Klotho reduces calciuria in distal tubules by its sialidase activity, acting directly on the apical calcium channel. Desialidation of the channel uncovers glycan residues that promote binding to galectin-1, resulting in stabilization of residence on the plasma membrane [25]. Further studies are needed to explain this correlation in $\mathrm{CKD}$ and dialysis patients. Also, more research is needed to assess the correlations between $\mathrm{Ca}$ and klotho in healthy subjects.

Although the current study provided new information regarding serum soluble klotho, our results should be interpreted in the context of some limitations. First, the size of study population was small making subgroup analysis difficult and therefore, multiple linear regression analyses may not be the most appropriate procedure. Second, although we tried to eliminate some medications that may affect $\mathrm{Ca}$ and $\mathrm{P}$ metabolism, the data regarding soluble klotho may be modified by some medications such as renin-angiotensin inhibitors, recombinant human erythropoietin and immunosuppressive treatments. Third, as we mentioned earlier, the available s-klotho assays measure soluble s-klotho that may not reflect the molecule in its membrane-bound state.

In conclusion, although FGF-23 requires its obligate co-receptor membrane-bound klotho for its actions on peripheral tissues, we have shown that circulating s-klotho was not related to FGF-23 in CKD, dialysis and KTR patients. Also, we have demonstrated a novel association between serum $\mathrm{Ca}$ and s-klotho, which requires further studies.

\section{References}

1 Kuro-o M, Matsumura Y, Aizawa $\mathrm{H}$, et al: Mutation of the mouse klotho gene leads to a syndrome resembling ageing. Nature 1997; 390:45-51.

2 Kuro-o M: Overview of the FGF23-Klotho axis. Pediatr Nephrol 2010;25:583-590.

3 Kuro-o M: Phosphate and Klotho. Kidney Int 2011;79:S20-S23.

4 Kurosu H, Ogawa Y, Miyoshi M, et al: Regulation of fibroblast growth factor-23 signaling by klotho. J Biol Chem 2006;281:61206123.
5 Wolf M: Update on fibroblast growth factor 23 in chronic kidney disease. Kidney Int 2012; 82:737-747.

-6 Seiler S, Wen M, Roth HJ, et al: Plasma Klotho is not related to kidney function and does not predict adverse outcome in patients with chronic kidney disease. Kidney Int 2012;83: 121-128.

7 Gutierrez O, Isakova T, Rhee E, Shah A, Holmes J, Collerone G, Juppner H, Wolf M: Fibroblast growth factor-23 mitigates hyperphosphatemia but accentuates calcitriol defi- ciency in chronic kidney disease. J Am Soc Nephrol 2005;16:2205-2215.

8 Koh N, Fujimori T, Nishiguchi S, Tamori A, Shiomi S, Nakatani T, Sugimura K, Kishimoto T, Kinoshita S, Kuroki T, Nabeshima Y: Severely reduced production of klotho in human chronic renal failure kidney. Biochem Biophys Res Commun 2001;280: 1015-1020.

9 Negri A: [FGF23 in chronic kidney disease and kidney post-transplant patients]. Nefrologia 2009;29:196-202. 
10 Ibrahim S, Rashed L: Serum fibroblast growth factor-23 levels in chronic haemodialysis patients. Int Urol Nephrol 2009;41:163-169.

-11 Bhan I, Shah A, Holmes J, Isakova T, Gutierrez $\mathrm{O}$, Burnett SM, Jüppner H, Wolf M: Post-transplant hypophosphatemia: tertiary 'hyper-phosphatoninism'? Kidney Int 2006;70:1486-1494.

12 Shigematsu T, Kazama JJ, Yamashita T, Fukumoto S, Hosoya T, Gejyo F, Fukagawa M: Possible involvement of circulating fibroblast growth factor 23 in the development of secondary hyperparathyroidism associated with renal insufficiency. Am J Kidney Dis 2004;44: 250-256.

13 Gutierrez O, Isakova T, Rhee E, Shah A, Holmes J, Collerone G, Jüppner $\mathrm{H}$, Wolf $\mathrm{M}$ : Fibroblast growth factor-23 mitigates hyperphosphatemia but accentuates calcitriol deficiency in chronic kidney disease. J Am Soc Nephrol 2005;16:2205-2215.

14 Sugiura H, Tsuchiya K, Nitta K: Circulating levels of soluble $a$-Klotho in patients with chronic kidney disease. Clin Exp Nephrol 2011;15:795-796.

15 Pavik I, Jaeger P, Ebner L, Wagner CA, Petzold K, Spichtig D, Poster D, Wüthrich RP, Russmann S, Serra AL: Secreted Klotho and FGF23 in chronic kidney disease Stage 1 to 5: a sequence suggested from a cross-sectional study. Nephrol Dial Transplant 2013;28:352-359.
16 Yokoyama K, Imura A, Ohkido I, Maruyama Y, Yamazaki Y, Hasegawa H, Urae J, Sekino H, Nabeshima Y, Hosoya T: Serum soluble a-Klotho in hemodialysis patients. Clin Nephrol 2012;77:347-351.

17 Akimoto T, Kimura T, Watanabe Y, Ishikawa $\mathrm{N}$, Iwazu Y, Saito O, Muto S, Yagisawa T, Kusano E: The impact of nephrectomy and renal transplantation on serum levels of soluble Klotho protein. Transplant Proc 2013;45: 134-136.

18 Viaene L, Bammens B, Meijers BK, Vanrenterghem Y, Vanderschueren D, Evenepoel P: Residual renal function is an independent determinant of serum FGF-23 levels in dialysis patients. Nephrol Dial Transplant 2012;27: 2017-2022.

19 Yamazaki Y, Imura A, Urakawa I, et al: Establishment of sandwich ELISA for soluble alpha-Klotho measurement: age-dependent change of soluble alpha-Klotho levels in healthy subjects. Biochem Biophys Res Commun 2010;398:513-518.

20 Wan M, Smith C, Shah V, Gullet A, Wells D, Rees L, Shroff R: Fibroblast growth factor 23 and soluble klotho in children with chronic kidney disease. Nephrol Dial Transplant 2013;28:153-161.
21 Leone F, Lofaro D, Gigliotti P, Perri A, Vizza D, Toteda G, Lupinacci S, Armentano F, Papalia T, Bonofiglio R: Soluble Klotho levels in adult renal transplant recipients are modulated by recombinant human erythropoietin. J Nephrol 2014;27:577-585.

22 Akimoto T, Kimura T, Watanabe Y, Ishikawa N, Iwazu Y, Saito O, Muto S, Yagisawa T, Kusano E: The impact of nephrectomy and renal transplantation on serum levels of soluble Klotho protein. Transplant Proc 2013;45: 134-136.

23 Sugiura H, Yoshida T, Mitobe M, Shiohira S, Nitta K, Tsuchiya K: Recombinant human erythropoietin mitigates reductions in renal klotho expression. Am J Nephrol 2010;32: 137-144.

24 Shibata K, Fujita S, Morita H, Okamoto Y, Sohmiya K, Hoshiga M, Ishizaka N: Association between circulating fibroblast growth factor 23, a-Klotho, and the left ventricular ejection fraction and left ventricular mass in cardiology inpatients. PLoS One 2013. 8:e73184.

25 Huang CL, Moe OW: Klotho: a novel regulator of calcium and phosphorus homeostasis. Pflugers Arch 2011;462:185-193.

$26 \mathrm{Hu}$ MC, Moe OW: Klotho as a potential biomarker and therapy for acute kidney injury. Nat Rev Nephrol 2012;8:423-429. 